\title{
Recommendation for an Oral Mucosa Contouring Method in Nasopharyngeal Carcinoma Patients receiving Tomotherapy
}

\author{
Peijing Li ${ }^{1}$, Yuanyuan Chen ${ }^{*}$ \\ 1 Department of Radiology, Zhejiang Cancer Hospital, Hangzhou, People's Republic of China \\ 2 Department of Radiation Oncology, Zhejiang Cancer Hospital, Hangzhou, People's Republic of China
}

\section{Background}

Radiotherapy is the primary treatment modality for non-disseminated nasopharyngeal carcinoma (NPC). Last two decades, intensitymodulated radiotherapy (IMRT) and volumetric-modulated arc therapy (VMAT) were widely used in many cancer centers around the world. Recently, tomotherapy (TOMO) has shown promising dosimetric advantages in the treatment of NPC, by delivery of better dose conformity in planning target volumes and sparing effect on organs at risk $^{(1)}$. Thus, this technique is increasingly used in NPC, especially for locally advanced disease. Radiation-induced oral mucositis (ROM) is not uncommon seen during the period of radiotherapy in NPCs, which may result in reduced quality of life (QOL) and affect patients' compliance to treatment ${ }^{(2)}$. There were a series of studies ${ }^{(3-6)}$ of head and neck cancer focused on relationship between ROM and dose parameters, however, with different radiation techniques, contouring methods and evaluation criterion of ROM, the results are varied and no consistent conclusions have been reached. Our previous studies ${ }^{(3,7)}$ revealed V30 (Vx, the percentage volume of the organ received more than $\mathrm{x} \mathrm{Gy)}$ as an independent predictor for ROM and also found no difference between contouring methods of OCC (oral cavity contour) and MSC (mucosa surface contour) by using IMRT. However, whether it is can be applied in TOMO is still unknown. Therefore, the aim of this study is to evaluate these two oral mucosa contouring methods in NPC patients receiving TOMO and find a reasonable contouring method for oral mucosa by using this new technique of radiotherapy.

\section{Methods}

A total of 125 American Joint Committee on Cancer (AJCC) 7th stage II-IVB NPC patients receiving TOMO in our center were included. Two contouring methods, the OCC (Fig 1) for the oral cavity and the MSC (Fig 2) for the mucosal surface, were applied to the radiation treatment plans. ROM were prospectively assessed weekly and recorded by two senior radiologists. Absolute DVH data was exported from the RayStation version 3.0 treatment planning system. T-test was used to compare all dosimetric parameters (Dmax [max dose], Dmean [mean dose] and V5-70 of OCC and MSC at five-unit intervals) between patients with $0-2$ grade and $\geq 3$ grade ROM. All of the significantly different parameters from the t-tests were separately included in the multivariate analysis. Binary logistic regression model was used to identify the most relevant parameters associated with $\geq 3$ grade ROM. Lastly, Receiver operating characteristic (ROC) analysis was used to carry out the cut-off value. $\mathrm{P}<0.05$ was considered significant.

Fig 1. OCC method

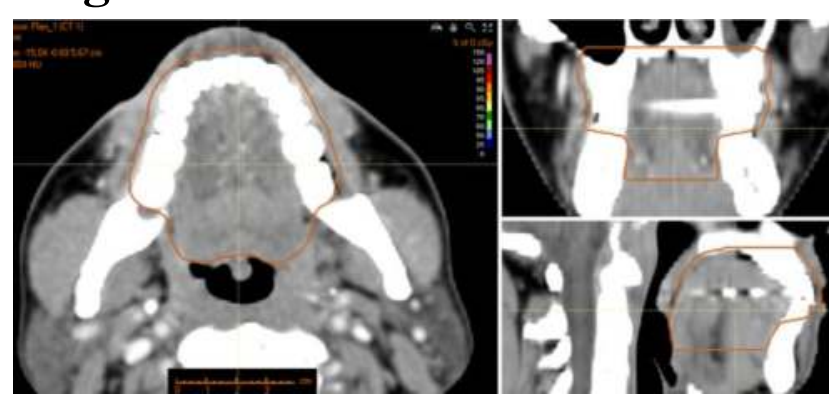

Fig 2. MSC method

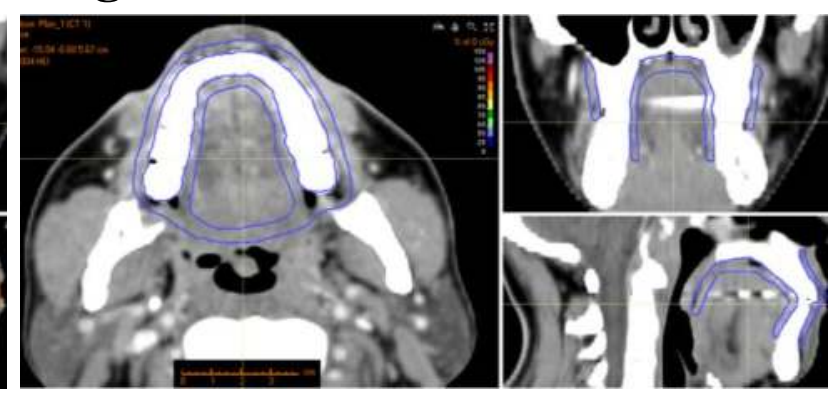

Table 1. Predictive factors by using methods of OCC and MSC in binary logistic regression analysis.

\begin{tabular}{lllll}
\hline Contouring methods & Predictive factors & & OR $(95 \% \mathrm{CI})$ & P value \\
\hline OCC & Gender & Male vs.Female & $0.060(0.011-0.311)$ & 0.001 \\
& Somking & Yes vs. No & $12.250(2.421-61.980)$ & 0.002 \\
MSC & Gender & Male vs.Female & $0.122(0.027-0.542)$ & 0.006 \\
& Somking & & $5.028(1.145-22.079)$ & 0.032 \\
& V55 & & $1.665(1.172-2.365)$ & 0.004 \\
\hline OCC: oral cavity contour; MSC: mucosa surface contour; CI: confidence interval ; OR: odds ratio \\
; V55: percentage volume of organ received more than 55 Gy & \\
\hline
\end{tabular}

\section{Results}

Grade $\geq 3$ acute ROM occurred in $34.4 \%$ (43/125) of patients in our study. Fig 3 showed the target dose distribution of MSC and OCC in a patient with grade 3 acute oral mucositis, some part of the area covered $5000 \mathrm{cGy}$ and $5500 \mathrm{cGy}$ were distributed in the oral area outside the oral mucosa. A significant dose-volume relationship between oral mucosa related dosimetric parameters and $\geq 3$ grade ROM were found by using both methods in univariate analysis: V10, V15, V45, V55, V60, V65, V70 of OCC and V55, V60, V65, Dmean of MSC were relevant dosimetric parameters for $\geq 3$ grade ROM (all P 0.05). In binary logistic regression analysis, gender, smoking were found significantly related to $\geq 3$ grade ROM by using OCC (male vs. female: $\mathrm{OR}=0.060$ 95\% CI $=0.011-0.311 ， \mathrm{P}=0.001$; smoking vs. non-smoking: $\mathrm{OR}=12.250 ， 95 \% \mathrm{CI}=2.421-61.980 ， \mathrm{P}=0.002$ ). For MSC, gender, smoking and MSC V55 were independent predictors (male vs. female $\mathrm{OR}=0.122 ， 95 \% \quad \mathrm{CI}=0.027-0.542 ， \mathrm{P}=0.006$; smoking vs. nonsmoking: $\mathrm{OR}=5.028 ， 95 \% \mathrm{CI}=1.145-22.079 ， \mathrm{P}=0.032$; MSC-V55 : $\mathrm{OR}=1.665,95 \% \mathrm{CI}=1.172-2.365, \mathrm{P}=0.004)$, as was shown in Table 1 . The cutoff of MSC-V55 was $9.58 \%$, area under curve was 0.698 , with sensitivity and specificity of 0.605 and 0.744 , respectively, as was shown in Fig 4.

Fig 3. Target dose distribution of MSC and OCC in a patient with grade 3 acute oral mucositis: partial doses of 5000cGy and 5500 cGy were distributed in the oral area outside the oral mucosa.
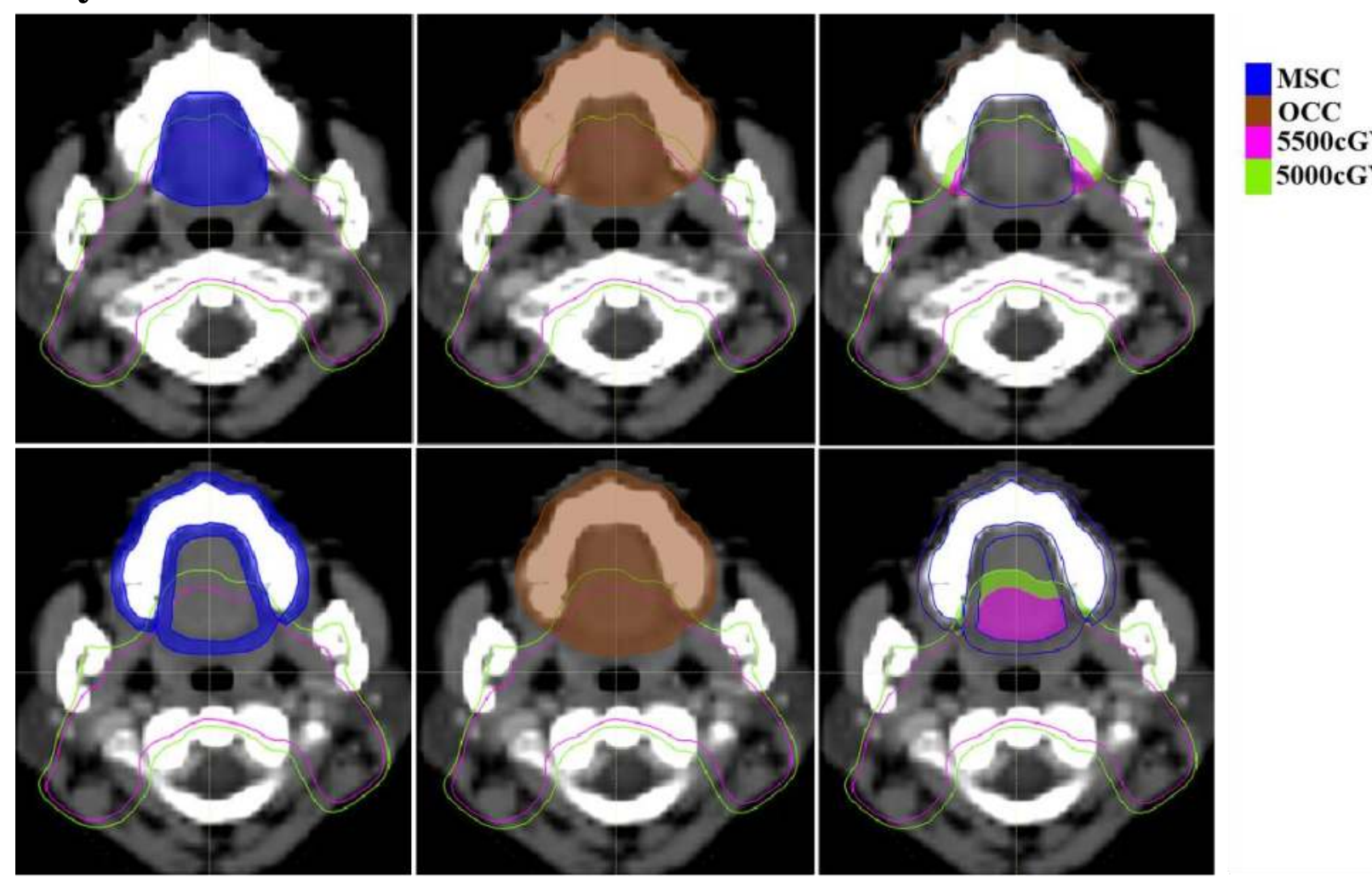
OCC 5000cGY

\section{Conclusion}

We recommend mucosa surface contour as a more reasonable contouring method for oral mucosa in tomotherapy treatment plan. However, AUC is not high enough to categorize the high risk case and the lower one. Data collection is continuing in our center, based on which, we want to further validate the results and find a more effective cut-off value.

\section{Reference}

1. Lee FK ho, Yip CW yi, Cheung FC hung, Leung AK cheung, Chau RM chun, Ngan RK cheong. Dosimetric difference amongst 3 techniques: TomoTherapy, sliding-window intensity-modulated radiotherapy (IMRT), and RapidArc radiotherapy in the treatment of late-stage nasopharyngeal carcinoma (NPC). Med Dosim [Internet]. 2014;39(1):44-9. Available from: http://dx.doi.org/10.1016/j.meddos.2013.09.004

2. Jin T, Li K, Li P, Huang S, Chen X. An evaluation of nutrition intervention during radiation therapy in patients with locoregionally advanced nasopharyngeal carcinoma. 2017;8(48):83723-33.

3. Li K, Yang L, Xin P, Chen Y, Hu Q-Y, Chen X-Z, et al. Impact of dose volume parameters and clinical factors on acute radiation oral mucositis for locally advanced nasopharyngeal carcinoma patients treated with concurrent intensity-modulated radiation therapy and chemoradiotherapy. Oral Oncol. 2017 Sep;72:32-7.

4. Wang ZH, Zhang SZ, Zhang ZY, Zhang CP, Hu HS, Tu WY, et al. Protecting the oral mucosa in patients with 4. Wang ZH, Zhang SZ, Zhang ZY, Zhang CP, Hu HS, Tu WY, et al. Protecting the oral mucosa in patients with
oral tongue squamous cell carcinoma treated postoperatively with intensity-modulated radiotherapy: A oral tongue squamous cell carcinoma treated postope
randomized study. Laryngoscope. 2012;122(2):291-8.

5. Mazzola R, Ricchetti F, Fersino S, Fiorentino A, Giaj Levra N, Di Paola G, et al. Predictors of mucositis in oropharyngeal and oral cavity cancer in patients treated with volumetric modulated radiation treatment: A dosevolume analysis. Head Neck. 2016;38:E815-9.

6. Musha A, Shimada H, Shirai K, Saitoh JI, Yokoo S, Chikamatsu K, et al. Prediction of acute radiation mucositis using an oral mucosal dose surface model in carbon ion radiotherapy for head and neck tumors. PLoS One. 2015;10(10):1-10

7. Li K, Yang L, Hu Q ying, Chen X zhong, Chen M, Chen Y. Oral Mucosa Dose Parameters Predicting Grade $\geq 3$ Acute Toxicity in Locally Advanced Nasopharyngeal Carcinoma Patients Treated With Concurrent IntensityAcute Toxicity in Locally Advanced Nasopharyngeal Carcinoma Patients Treated With Concurrent Intensity-
Modulated Radiation Therapy and Chemotherapy: An Independent Validation Study Comparing Oral Cavity vers. Transl Oncol [Internet]. 2017;10(5):752-9. Available from: https://doi.org/10.1016/j.tranon.2017.06.011 